\title{
Influence des actions anthropiques sur la dynamique spatio-temporelle de l'occupation du sol dans la province du Bas-Congo (R.D. Congo).
}

\author{
Issouf Bamba ${ }^{1 *}$, Adi Mama ${ }^{2}$, Danho F. R. NeUBA ${ }^{3}$, Kouao J. KoffI ${ }^{1}$, Dossahoua TraORÉ ${ }^{4}$, Marjolein VISSER ${ }^{1}$, Brice SINSIN ${ }^{1}$, \\ Jean LEJOLY' \& Jan BOGAERT ${ }^{1}$

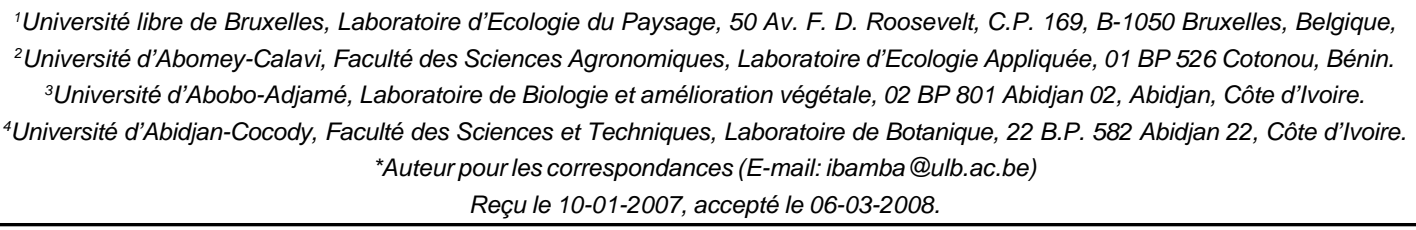

\section{Résumé}

Située à proximité de Kinshasa, la province du Bas-Congo (R.D. Congo) est l'une des principales pourvoyeuses en produits vivriers de cette ville. Par conséquent, l'augmentation de la pression sur les ressources naturelles s'accentue. L'analyse diachronique du paysage peut servir à montrer l'effet des actions anthropiques sur l'occupation du sol de cette province. La présente étude a pour but la quantification de la dynamique paysagère dans une zone test de cette province entre 1960 et 2005. Elle a permis, grâce à la matrice de transition appuyée par le calcul d'indices de structure spatiale, de montrer que l'occupation du sol a profondément changé. La matrice du paysage, initialement constituée par les forêts secondaires (49,95\% du paysage), s'est dégradée au profit des savanes (qui ont conquis $14,23 \%$ des forêts secondaires) et des jachères et champs (qui ont conquis $27,23 \%$ des forêts secondaires). L'étude a ainsi révélé trois processus de transformation du paysage: la savanisation, la fragmentation de l'écosystème forestier et enfin une la formation de forêt dense (succession). Ces changements - excepté la succession - sont principalement dus à des perturbations anthropiques. La pression démographique et les pratiques agricoles non durables ont orienté la modification de l'occupation du sol. Les écosystèmes forestiers ont été substitués par des écosystèmes anthropisés menaçant alors la biodiversité de cette zone.

Mots clés: Dynamique paysagère, occupation du sol, pression anthropique, système agraire, Province du Congo central, biodiversité.

\section{Abstract \\ Impact of human activity on the spatio-temporal dynamics of land cover in the Bas-Congo province (D.R. Congo)}

Situated nearby Kinshasa, the Bas-Congo province (Democratic Republic of the Congo) is one of the principal providers of food products of this city. Consequently, an increased anthropogenic pressure on natural resources is observed. Analysis of the landscape dynamics can be used to demonstrate the impact of human activities on land cover in this province. The objective of this study is to quantify landscape dynamics between 1960 and 2005 in a test zone situated in the aforementioned province Based on the interpretation of the transition matrix and on the calculation of spatial pattern metrics, a profound change of land cover has been shown. The landscape matrix, initially constituted by secondary forests (49.95\% of the landscape), has been degraded and replaced by savannah vegetation (substitution of $14.23 \%$ of the secondary forests) and mosaics of fallow land and fields (substitution of $27.23 \%$ of the secondary forests). Three spatial processes of land transformation have been identified: creation of savannah, forest degradation or fragmentation, and formation of dense forest (succession). These changes - except succession - are mainly due to anthropogenic disturbance. Demographic pressure and unsustainable agricultural practices have contributed to the change of land cover. Forest ecosystems are substituted by anthropogenic ecosystems which indicates a threat to the biodiversity of this area.

Key words: Landscape dynamics, land cover, anthropogenic pressure, agricultural system, Central Congo Province, biodiversity. 


\section{Introduction}

Les ressources naturelles subissent des pressions anthropiques croissantes qui entraînent des dysfonctionnements des écosystèmes terrestres et des pertes de biodiversité (Roche, 1998). Encore amplifiés par les modes et systèmes inappropriés d'exploitation des ressources disponibles, ces changements ont des répercussions directes sur l'occupation du sol et sur la configuration du paysage. Les processus naturels de succession des végétations sont alors perturbés par l'activité anthropique à travers l'exploitation du bois d'œuvre et les diverses techniques culturales, principalement l'agriculture itinérante (Vink, 1983).

Le paysage, espace géographique composé d'un ensemble d'écosystèmes en interaction, est dynamique (Bogaert \& Mahamane, 2005). La compréhension de cette dynamique spatiotemporelle est cruciale en raison des interactions avec les activités humaines (Schlaepfer, 2002). La dynamique paysagère pourrait ainsi être mise en évidence et quantifier par l'analyse de la composition et la configuration de ses éléments. La structure spatiale des écosystèmes paysagers peut donc contribuer à éclairer les processus écologiques qui s'y déroulent (Fortin, 2002). En effet, chaque système écologique est caractérisé par une interdépendance de trois éléments clés: sa structure spatiale, sa composition et son fonctionnement (Bogaert \& Mahamane, 2005). De par cette relation, le paysage sera directement lié à la biodiversité et illustrera la confrontation qui existe entre la société et son milieu (Burel \& Baudry, 2003).

En République Démocratique du Congo (R.D. Congo), environ $60 \%$ de la population vit en milieu rural (FAO, 2001). Après Kinshasa (la capitale) et la province du Nord Kivu, le BasCongo (ex-Bas-Congo) est la troisième province la plus dense avec environ 52 habitants $/ \mathrm{km}^{2}$ inégalement repartie et concentrée dans les principales villes que sont Matadi et Boma (Tshibangu, 2001). Il a été observé une intensification de la pression anthropique sur les ressources naturelles ainsi qu'une surexploitation des sols due au déboisement systématique le long de grands axes routiers et à des mauvaises pratiques culturales (Bizangi, 2004). De nombreuses études au niveau de la région ont montré un recul des forêts tropicales humides sans pour autant quantifier véritablement ce phénomène. Les travaux de Tshibangu (2001) basés sur une étude cartographique du déboisement entre 1960 et 1987 révélaient un fort recul forestier dans la zone de Kinshasa. De par la proximité (moins de 100 $\mathrm{km}$ ), le Bas-Congo (ex-Bas-Congo) constitue la principale entité pourvoyeuse en produits vivriers de la capitale (Tshibangu, 2001). Par conséquent, la pression sur les ressources naturelles ne cesse de s'accentuer. C'est pourquoi le suivi et la quantification de la dynamique de l'occupation du sol dans cette zone s'avère nécessaire pour attirer l'attention sur ces paysages fortement dépendants des pratiques culturales ancestrales de la population.

Notre étude a pour but de montrer et de quantifier, à partir de données diachroniques (cartes d'occupation du sol géoréférencées de 1960 et de 2003) et des techniques de l'écologie du paysage, la dynamique dans le temps et dans l'espace de l'occupation du sol d'une zone test de la province du Bas-Congo.

\section{Matériel et méthodes}

\subsection{Description de la zone d'étude}

La zone étudiée est située dans la province du Bas-Congo en R.D. Congo, entre $15^{\circ} 23^{\prime}$ et $15^{\circ} 38^{\prime}$ de longitude Est et entre $4^{\circ} 52^{\prime}$ et $5^{\circ} 00^{\prime}$ latitude Sud (Fig. 1). Cette zone d'une superficie d'environ $410 \mathrm{~km}^{2}$ est localisée à moins de $100 \mathrm{~km}$ de la grande métropole Kinshasa (15⒉ $\left.24^{\prime} \mathrm{E}, 4^{\circ} 24^{\prime} \mathrm{S}\right)$ et à moins de $50 \mathrm{~km}$ de la route Nationale 1, la principale voie d'accès au port de Matadi (13⒉ $\left.27^{\prime} E, 5^{\circ} 50^{\prime} S\right)$. Le climat est du type $A w_{4}$ de Köppen avec une saison sèche marquée de juin à septembre, la température moyenne de $25^{\circ} \mathrm{C}$ et des précipitations moyennes annuelles variant entre 900 et $1500 \mathrm{~mm}$. Le relief est très varié dominé par des plateaux avec une altitude maximale de $750 \mathrm{~m}$. La végétation, est hétérogène. Elle est dominée par les formations végétales de type savanicole malgré un climat favorable aux formations forestières (Compère, 1970). 


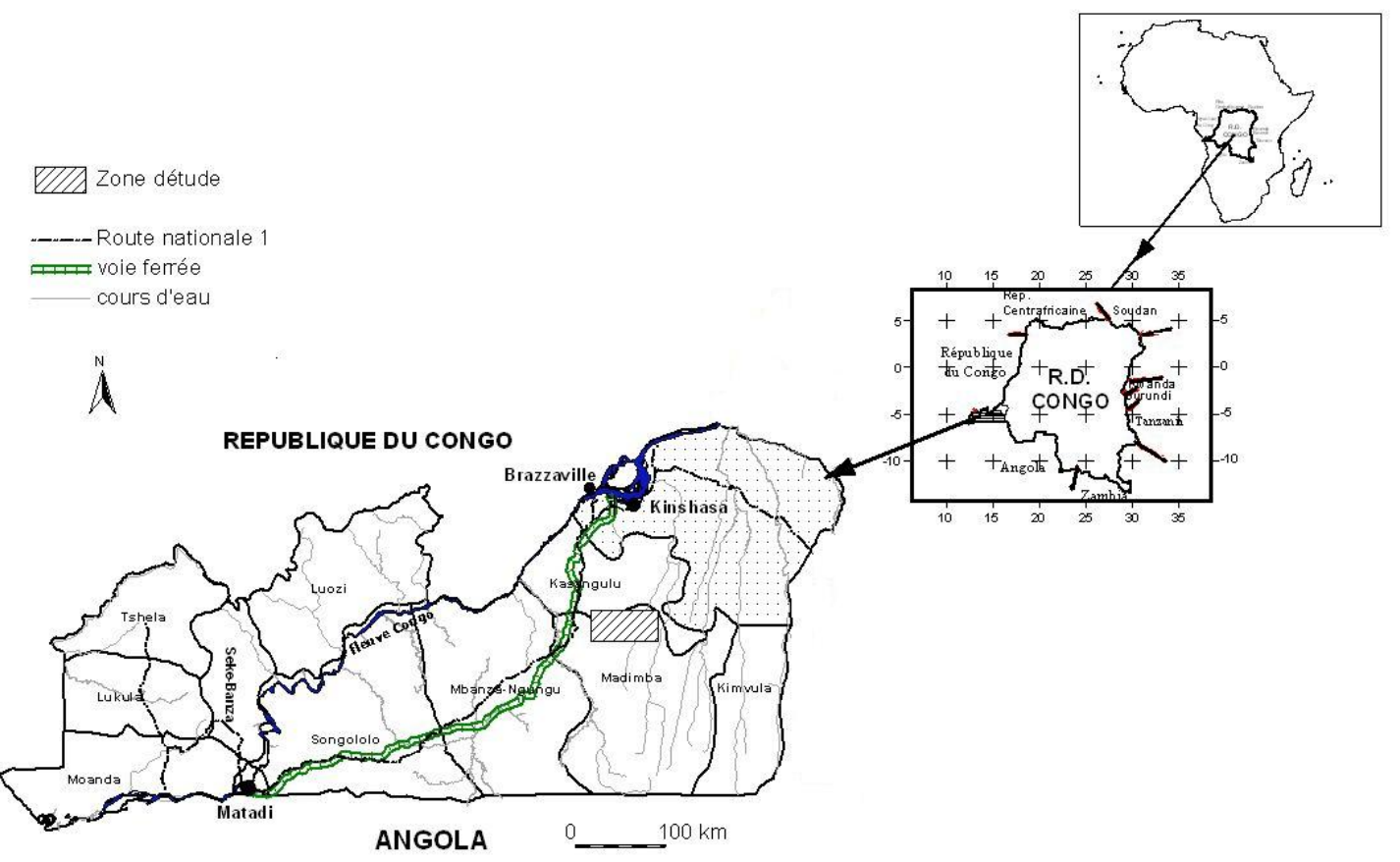

Figure 1: Situation de la zone d'étude dans la province du Bas-Congo (R.D. Congo). Source des données vectorielles : Université catholique de Louvain, Unité d'environnemétrie et de géomantique.

\subsection{Matériel}

La dynamique du paysage peut être décrite par des changements dans le temps de sa composition et de sa structure spatiale. L'application des méthodes de l'écologie du paysage (calcul d'indices de structure spatiale) est basée sur la relation d'interdépendance entre les trois éléments clés de tout système écologique à savoir sa configuration spatiale, sa composition et son fonctionnement (Bogaert \& Mahamane, 2005). Généralement, cela nécessite l'usage de données de télédétection (images satellitaires et photographies aériennes) et les cartes thématiques. Dans le cas présent, nous avons eu recours à deux cartes d'occupation du sol de la province du Bas-Congo. La première carte a été conçue par Compère (1970) à l'échelle de 1:250 000 par réduction de cartes minutes au 1:200 000 grâce à l'interprétation de photographies aériennes prises en 1959 et 1960 par I'Institut Géographique du Congo et complétée par des prospections de terrain. Elle présente dix classes d'occupation du sol
(Tableau 1). La seconde carte (Wolff, 2005) a été produite à l'échelle de 1:75 000 par le traitement d'images ASTER (du 18/07/2003) complétées par des levées de terrain en 2005. Cette carte présente quatre classes d'occupation du sol (Tableau 1). Les logiciels de SIG et de traitements cartographiques utilisés sont: Arcview 3.3 ; Idrisi kilimandjaro.

\subsection{Méthodes}

La présente étude s'inscrivait dans le cadre d'une caractérisation de la dynamique de l'occupation du sol entre 1960 et 2005 dans un paysage rural. Pour ce faire, les transformations d'occupation du sol opérés entre ces dates ont été quantifiées à l'aide de la matrice de transition et d'indices de structure spatiale afin d'évaluer l'influence des techniques culturales sur ce paysage.

\subsubsection{Homogénéisation des légendes}

Le traitement cartographique des données a été le premier volet de la méthodologie de notre étude. II a consisté dans un premier temps à la 
digitalisation de la carte de 1960 (Compère 1970) qui était en format papier contrairement à celle de 2005 (Wolff 2005) qui était déjà en format numérique. La numérisation s'est faite avec le logiciel Arcview 3.3. Puis il fallait homogénéiser les légendes. Cette homogénéisation a été indispensable car elle a permis de compenser les inégalités entre les classes des légendes en regroupant certaines classes (Ozenda, 1986). Ainsi, nous avons préalablement réduit la légende de la carte de Compère (1970) par fusion de certaines classes, afin d'avoir les mêmes quatre classes de la carte de Wolff (2005) pour les deux cartes (Tableau 1). Ensuite la technique du Minimum Mapping Unit (Saura, 2002) a permis de mettre la précision cartographique des deux cartes au même niveau. L'extension "Dissolve by area " du logiciel Arcview 3.3, a permis suivant cette méthode de fusionner les taches dans la carte de Wolff (2005) de sorte que la plus petite tache sur cette carte ait la même superficie que la plus petite tache repérée dans la carte de Compère (1970).

Tableau 1 : Correspondance des différentes classes entre les cartes de Wolff (2005) et Compère (1970).

\begin{tabular}{lll}
\hline \multicolumn{2}{c}{ Classes de Wolff (2005) } & \multicolumn{1}{c}{ C Classes de Compère (1970) } \\
\hline \multirow{2}{*}{ 1. Savane } & 1. & Savanes mésophiles sur sols lourds \\
& 2. & Savanes xérophiles sur sols lourds \\
& 3. & Savanes mésophiles sur sols légers \\
& 4. & Steppes et savanes steppiques sur sols légers \\
\hline \multirow{2}{*}{ 2. Jachères et champs } & 5. & Recrus et jachères forestières \\
& 6. & Végétation nitrophile et postculturale \\
\hline \multirow{2}{*}{ 3. Forêt secondaire } & 7. & Forêts guinéennes et périguinéennes secondarisées \\
& 8. & Forêts de reconstitution et vieilles forêts secondaires \\
\hline \multirow{2}{*}{ 4. Forêt dense } & 9. & Forêts denses humides semi-décidues subéquatoriales et guinéennes \\
\hline
\end{tabular}

\subsubsection{Matrice de transition de l'occupation du sol}

Pour décrire les changements d'occupation du sol intervenus entre 1960 et 2005, la méthode de la matrice de transition a été utilisée. La matrice de transition entre deux états ( $t_{0}$ et $t_{1}$ ) est obtenue à partir des valeurs données par les logiciels de SIG et traitées dans Excel. Ces valeurs proviennent de la superposition des deux cartes grâce à un logiciel de SIG (ici l'extension Geoprocessing Wizard de Arcview 3.3) en vue de détecter les changements opérés dans l'occupation du sol entre deux dates. Elle correspond à une matrice carrée décrivant de manière condensée, les changements d'état des éléments d'un système pendant une période donnée (Schlaepfer, 2002). Les cellules de la matrice contiennent la valeur d'une variable ayant passé d'une classe initiale $i$ à une classe finale $j$ pendant la période allant de $t_{0}$ à $t_{1}$. Les valeurs des colonnes et des lignes représentent des proportions des aires occupées par chaque classe d'occupation du sol au temps correspondant. Ainsi, les colonnes de la matrice indiquent les états d'occupations des sols en 2005 et les lignes correspondent aux états en 1960.

\subsubsection{Calcul d'indice de structure spatiale}

La suite de l'analyse a consisté au calcul d'un certain nombre d'indices de structure spatiale. II est admis que le paysage est composé par trois types d'éléments: (1) les taches, entités élémentaires fonctionnelles d'une classe d'occupation du sol, (2) les corridors, éléments linéaires reliant les taches entre elles et (3) la matrice, l'élément englobant qui exerce le rôle dominant (Burel \& Baudry, 2003). Ainsi, afin d'étudier les rapports entre la configuration du paysage et les processus écologiques, il est nécessaire de décrire ces structures en termes quantifiables. Ceci explique le développement d'une série d'indices "landscape metrics» (Hargis et al., 1997). Ces mesures sont souvent un indicateur de l'impact humain sur la 
morphologie du paysage (Krummel et al., 1987). Dans le cadre de cette étude, un certain nombre d'indices au niveau de chaque classe d'occupation du sol ont été calculés.

Premièrement, le nombre de taches appartenant à une classe donnée $j\left(n_{j}\right)$ a été déterminé. Cet indice nous a renseigné sur la fragmentation d'une classe entre deux périodes. En effet, l'augmentation du nombre de taches d'une classe peut être due à la fragmentation de cette classe (Davidson, 1998).

L'aire totale $\left(a_{t j}\right)$ occupée par la classe $j\left(\mathrm{en} \mathrm{km}^{2}\right)$ a été calculée suivant l'équation (1) où $a_{i j}$ était l'aire de la $i$-ème tache de la classe $j$ :

$$
a_{i j}=\sum_{i=1}^{n_{i}} a_{i j}
$$

La dominance $D_{j}(a)$ indiquant la proportion d'aire occupée par la tache dominante dans la classe $j$ a aussi été prise en compte. II s'agit de la part occupée dans l'aire totale $\left(a_{t i}\right)$ par la plus grande tache de la classe jnotée $a_{\operatorname{maxj}}$ (McGarigal \& Marks, 1995):

$$
D_{j}(a)=\frac{a_{\max , j}}{a_{b j}} \times 100
$$

$0<D_{i}(a) \leq 100$. Plus la valeur de la dominance est grande, moins la classe est fragmentée. L'aire moyenne $\bar{a}_{i}$ (la valeur moyenne de l'aire des taches de la classe $j$ ) a été calculée selon la formule suivante :

$$
\overline{a_{j}}=\frac{a_{t_{j}}}{n_{j}}
$$

La diversité des aires des taches de la classe $j$, notée $H_{i}(a)$, a été calculée par l'indice de Shannon (Bogaert \& Mahamane, 2005). L'indice de diversité de Shannon est donné par la formule (4) où In représente le logarithme népérien :

$$
H_{j}(a)=\sum_{i=1}^{n_{j}}-\left(\frac{a_{i j}}{a_{i j}} \ln \frac{a_{i j}}{a_{i j}}\right)
$$

Cet indice mesure la diversité relative des taches au niveau de la classe. La valeur de $H_{j}(a)$ va dépendre du nombre de taches présentes $\left(n_{j}\right)$, de leurs proportions relatives $\left(\frac{a_{i j}}{a_{t j}}\right)$ et de la base du logarithme. II est égal à 0 lorsque la classe n'est constituée que d'une seule tache et sa valeur va croître avec le nombre de taches et avec l'équitabilité entre les aires des taches de la classe (McGarigal \& Marks, 1995).

L'indice de forme de la classe $j\left(I F_{j}\right)$ a été donnée par la formule (5) :

$$
I F_{j}=\frac{p^{2}{ }^{2}}{a_{t j}} \quad \text { (5) où } P_{i j} \text { était le périmètre total de }
$$

la classe $j$.

L'indice des formes est basé sur un principe de rapport du périmètre sur l'aire. La forme est un élément difficile à quantifier et qui peut donner libre cours à différentes interprétations (Ducrot, 2005). Elle peut être liée à des degrés d'artificialisation et des pratiques culturales (Krummel et al., 1987). Par exemple, les terres agricoles sont très polygonales alors que les formations naturelles, telles les forêts ont souvent un contour plus complexe. Elle est également liée à l'hétérogénéité du paysage (Delcros, 1994). Plus les taches ont des formes allongées ou irrégulières, plus la valeur de $I^{\prime} I F_{j}$ sera élevée et cette valeur décroîtra à mesure que les formes deviennent circulaires (Bogaert et al., 2000).

\section{Résultats}

\subsection{Transformations de l'occupation du sol entre 1960 et 2005}

Le tableau 2 donne les pourcentages de changements opérés entre les différentes classes d'occupation du sol entre 1960 et 2005 dans la zone test. Pour rappel, la superficie de la zone étant d'environ $410 \mathrm{~km}^{2}, 1 \%$ correspond alors à environ $4,1 \mathrm{~km}^{2}$. On constate que le taux de savane dans le paysage est passé de 19,81\% en 1960 à 29,61\% en 2005. Le taux de jachères et champs est passé de $22,72 \%$ en 1960 à $54,61 \%$ en 2005 ; cette classe constitue la nouvelle matrice du paysage précédemment constituée par la classe forêt secondaire. La présence de cette dernière classe qui était de 49,95\% en 1960 est tombée à 5,67\% en 2005. Quant à la forêt dense, son occupation du paysage est passé de $7,52 \%$ en 1960 à 10,10\% en 2005. De façon générale, on constate que les taux d'occupation des classes savanes, jachères et champs et forêt dense ont augmenté entre 1960 et 2005. Cette augmentation s'est principalement 
opérée au détriment de la forêt secondaire. Sur les $29,61 \%$ du paysage occupés par la savane en $2005,14,23 \%$ étaient de la forêt secondaire en 1960. De même, on observe pour les jachères et champs, que jusqu'à $27,32 \%$ sont issues de la forêt secondaire. Pour la classe forêt dense, $5,62 \%$ proviennent de l'évolution de la forêt secondaire. La classe forêt secondaire constitue donc la principale pourvoyeuse en espace des autres classes. On a ainsi environ $41,55 \%$ du paysage occupés par la forêt secondaire en 1960 qui s'est dégradé soit en savane, soit en jachères et champs. Le reste a évolué en forêt dense $(5,62 \%)$ ou est resté forêt secondaire $(2,78 \%)$.

Tableau 2 : Matrice de transition de l'occupation du sol (en pourcentage) entre 1960 et 2005.

\begin{tabular}{lccccc}
\hline \multirow{2}{*}{2005} & Savane & Jachères et champs & Forêtsecondaire & Forêt dense & Totaux \\
\hline Saúane & 9,30 & 8,89 & 0,71 & 0,91 & 19,81 \\
\hline Jachères et champs & 4,86 & 13,11 & 1,91 & 2,84 & 22,72 \\
\hline Forêt secondaire & 14,23 & 27,32 & 2,78 & 5,62 & 49,95 \\
\hline Forêt derse & 1,23 & 5,29 & 0,27 & 0,73 & 7,52 \\
\hline Totaux & 29,62 & 54,61 & 5,67 & 10,10 & 100 \\
\hline
\end{tabular}

En résumé, on observe trois grands processus qui se sont déroulés dans le paysage en 45 ans (Fig. 2, 3 et 4). Premièrement la savanisation, c'est-à-dire la création ou formation de savanes; elle s'est faite par le maintien des savanes préexistantes $(9,30 \%)$ et surtout par la disparition de la classe forêt secondaire
(14.23\%). Deuxièmement la dégradation de l'écosystème forestier, surtout de la forêt secondaire $(-22.63 \%)$. Finalement la succession naturelle spontanée : c'est l'évolution normale vers un état climacique. II s'agit de la restauration et formation de forêts $(+1.85 \%)$.

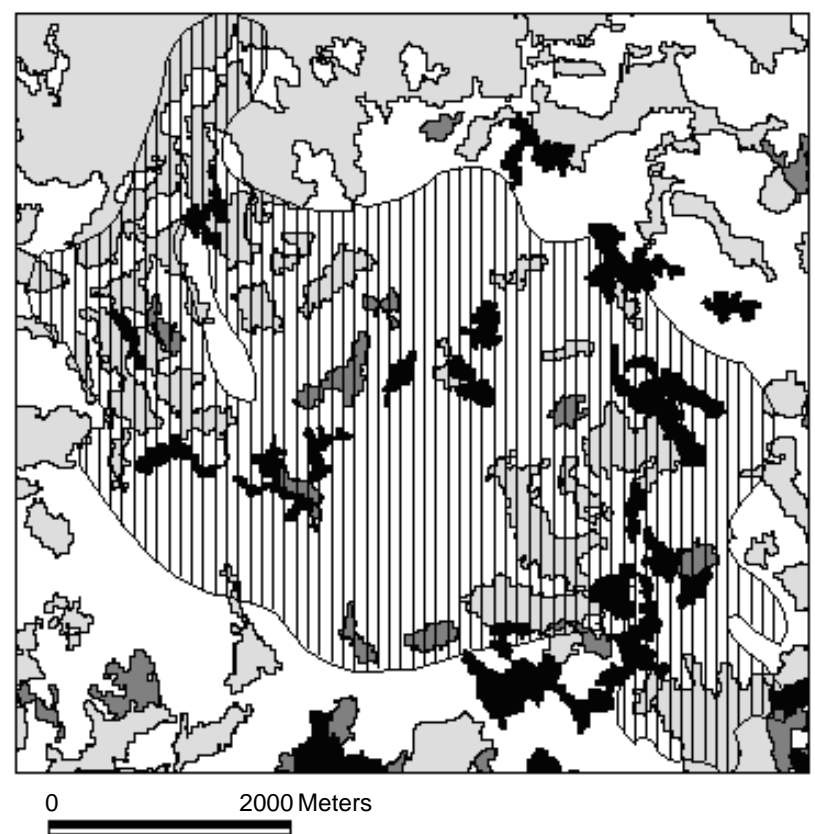

$\mathbf{N}$

Occupation du sol en 1960

ШाD Forêt dense

$\square$ autres

Occupation du sol en 2005

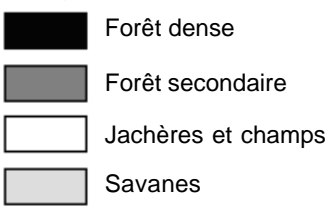

Figure 2: Illustration du processus de dégradation de la forêt dense entre 1960 et 2005. 

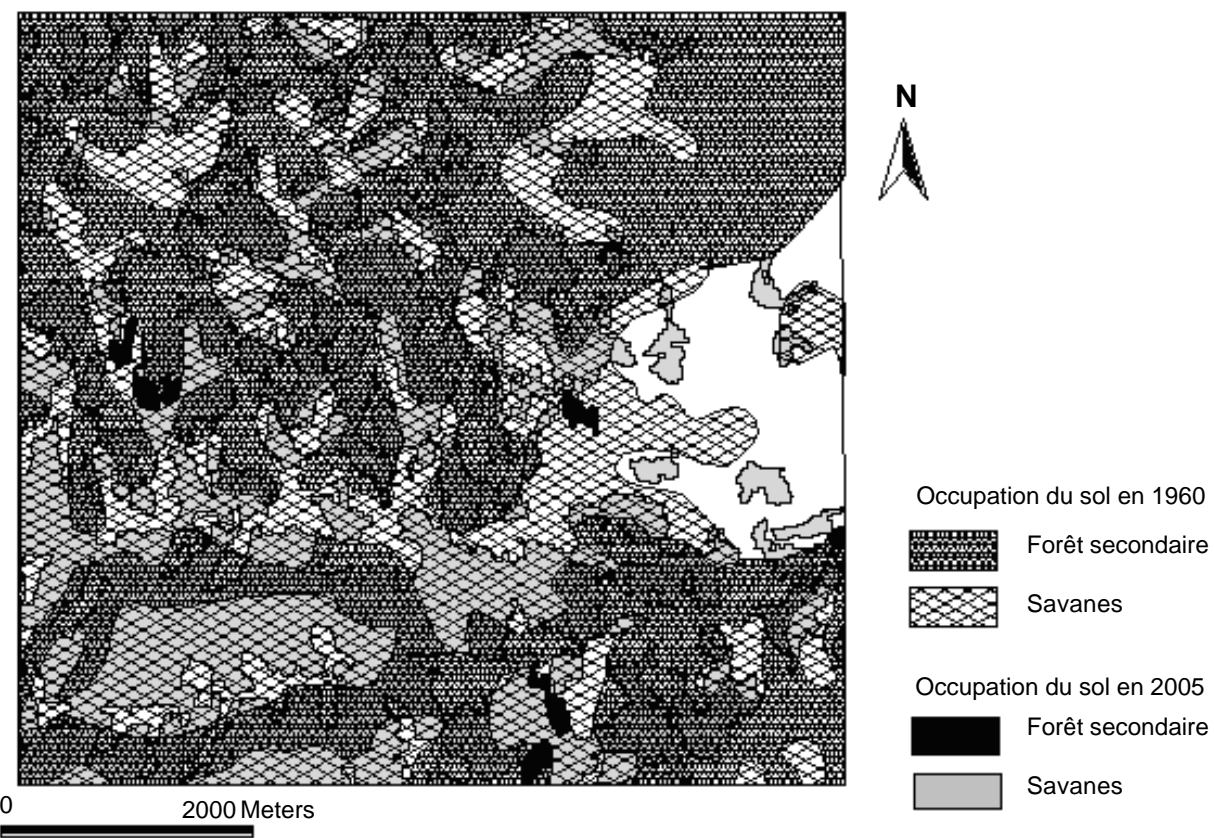

Figure 3 : Illustration du processus de savanisation de la forêt secondaire entre 1960 et 2005.

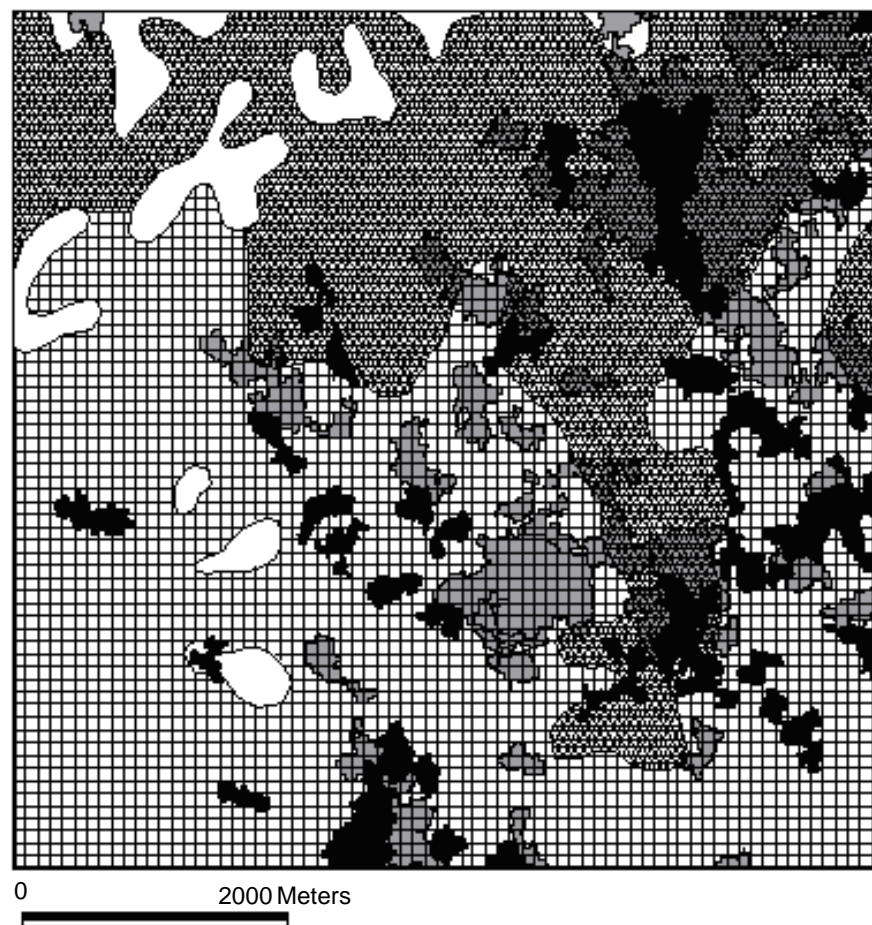

N

Occupation du sol en 1960

門器筑 Forêt secondaire

姍曲 Jachères et champs

Occupation du sol en 2005

$\square$ Forêt dense
$\square$ Forêt secondaire

Figure 4 : Illustration du processus de succession de jachères et champs et des forêts de 1960 à 2005. 


\subsection{Analyse de la dynamique de la structure spatiale}

Le tableau 3 récapitule les différents indices de structure spatiale calculés dans chacune des quatre classes en 1960 et en 2005. Ces indices ont permis de détecter les changements de la structure spatiale de notre paysage entre les deux dates. Le nombre de taches augmente entre 1960 et 2005 ; ceci indique une fragmentation avec un morcellement des taches initiales. Mais elle est de degrés variables. Pour la classe des savanes, le nombre de taches de 1960 a pratiquement triplé; pour les jachères et champs, il est multiplié par environ 10. En revanche, c'est dans les classes des forêts que la variation est la plus grande avec une multiplication par environ 50 et par environ 100 au niveau des forêts denses et secondaires respectivement. Cette tendance de fragmentation est confirmée par la diminution des aires moyennes dans l'ensemble des classes. En effet entre 1960 et 2005, pour les classes savane et jachères et champs, l'aire moyenne est respectivement divisée par environ 2 et par environ 5. Au niveau des classes des forêts (denses et secondaires), les valeurs des aires moyennes chutent considérablement. Dans la classe forêt secondaire, la valeur de cet indice est divisée par environ 1000 pendant que dans la classe forêt dense cet indice de 1960 est divisé par environ 35 (Tableau 3). Les valeurs de la dominance $\left(D_{j}(a)\right)$ diminuent pour ces mêmes classes passant de $99,69 \%$ à $10,95 \%$ dans les forêts secondaires et de $64,64 \%$ à $22,95 \%$ pour les forêts denses font aussi constater que ce sont ces deux classes qui sont les plus fragmentées. En effet, cela signifie qu'en 45 ans, les taches qui dominaient ces classes par leurs tailles ont été morcelées environ 10 fois pour les forêts secondaires et environ 3 fois pour les forêts denses. En revanche, on note que la valeur de la dominance augmente pour les classes des savanes $(10,30 \%$ à $23,68 \%)$ et aussi des jachères et champs $(47,81 \%$ à $80,78 \%)$. II y a donc eu formation ou agrégation de taches dans ces deux classes. L'indice de Shannon nous renseigne sur l'homogénéité au sein des classes. Plus cet indice est élevé, plus il y a de taches et sa valeur décroît à mesure que les aires des taches sont disproportionnelles. On constate que c'est aussi au niveau des forêts (denses et secondaires) que la valeur de l'indice augmente (environ 200 fois au niveau des forêts secondaires et environ 4 fois pour les forêts denses) alors que dans les classes savanes et jachères et champs, elle reste plutôt constante. Cela confirme la tendance d'équitabilité entre les taches de forêts dans lesquelles beaucoup de petites taches ont remplacé les quelques grosses présentes en 1960 comme déjà montré par les valeurs de $D_{j}$ (a). Un autre constat est l'évolution de l'indice des formes $I F_{i}$ avec les classes. On constate que pour toutes les classes où le taux d'occupation du sol augmente, la valeur de l'indice de forme augmente. Au niveau de la savane cette valeur passe de 36,31 en 1960 à 96,94 en 2005, dans les jachères et champs elle est passée de 43,78 à 188,77 et représente la plus grande valeur des indices de formes des classes de 2005; quant à la forêt secondaire, elle voit la valeur de cet indice diminuer de 821,9 en 1960 à 74,91 en 2005.

Tableau 3 : Indices de structures spatiales calculés en 1960 et 2005 pour chaque classe d'occupation du sol.

\begin{tabular}{|c|c|c|c|c|c|c|c|c|c|c|c|c|}
\hline & \multicolumn{2}{|c|}{$n_{j}$} & \multicolumn{2}{|c|}{$a_{j j}\left(\mathrm{~km}^{2}\right)$} & \multicolumn{2}{|c|}{$\bar{a}_{j}\left(\mathrm{~km}^{2}\right)$} & \multicolumn{2}{|c|}{$D_{j}(\%)$} & \multicolumn{2}{|c|}{$H_{j}(a)$} & \multicolumn{2}{|c|}{$\overline{I F}$} \\
\hline & 1960 & 2005 & 1960 & 2005 & 1960 & 2005 & 1960 & 2005 & 1960 & 2005 & 1960 & 2005 \\
\hline Savanes & 96 & 299 & 81,29 & 119.48 & 0,85 & 0.4 & 10,3 & 23,68 & 3,91 & 3,95 & 36,31 & 96,9 \\
\hline $\begin{array}{l}\text { Jachères et } \\
\text { champs }\end{array}$ & 14 & 169 & 105,26 & 220,39 & 7,52 & 1,3 & 47.81 & 80,78 & 1,84 & 1,3 & 43,78 & 188.7 \\
\hline $\begin{array}{l}\text { Forêt } \\
\text { secondaire }\end{array}$ & 2 & 212 & 201,98 & 22,86 & 100,96 & 0,11 & 99,69 & 10,95 & 0,02 & 4,71 & 821,9 & 74,91 \\
\hline F orêt dense & 4 & 195 & 30.27 & 40,79 & 7.57 & 0,21 & 64,64 & 22,95 & 1,04 & 4,09 & 27.6 & 85,37 \\
\hline
\end{tabular}




\section{Discussion}

\subsection{Influence des pratiques culturales sur les processus d'évolution du paysage}

Dans le processus de succession spontanée de la végétation, la phase forêt secondaire occupe une position centrale (Fig.5). Ce sont en effet, les forêts secondaires qui reconstitueront la forêt dense. Elles constituent le passage obligé vers l'état climacique dans un écosystème forestier (OIBT, 2002). La baisse de leur taux est un signe de perturbation pour la restauration de l'écosystème. Cette situation est due à une pratique non durable du système agraire, ellemême provoquée dans le cas de notre zone d'étude par une pression démographique de plus en plus croissante et par la réduction des temps de jachères (Whitmore, 2005). En effet, l'effectif de la population de cette région est passé de 1992 845 habitants en 1984 à 2835000 habitants en 1998 (Tshibangu, 2001). De plus, l'agriculture traditionnelle, dans la plupart des régions d'Afrique, est la culture itinérante. Cependant, à partir du moment où la densité de population atteint et dépasse certaines limites critiques, la période de jachère se raccourcit, et la végétation se dégrade, souvent irréversiblement (Kio, 1984). Cette agriculture itinérante sur brûlis serait responsable pour $70 \%$ de la déforestation en Afrique (Tshibangu, 2001). C'est exactement ce qui se passe dans la province du Bas-Congo. Les systèmes traditionnels basés sur la régénération naturelle ne fonctionnent plus comme jadis. En 45 ans, la matrice du paysage est passée de forêt secondaire aux jachères et champs. Ce constat confirme les études de Tshibangu (2001) menée dans la zone de Kinshasa. II a observé entre 1960 et 1987, une régression du couvert forestier et une progression de la zone urbaine (jachère et champs) et de la savane. Les savanes brûlées puis laissées pour compte se maintiennent dans les mêmes endroits tout en augmentant leur surface suite à la dégradation de certaines jachères. Selon Duvigneaud (1949), le climax de cette région est forestier. Les déboisements considérables, suivis d'une savanisation qui aurait tendance à se stabiliser sous l'action des feux, seraient responsables de la transformation du paysage ligneux primitif en paysage herbeux anthropogène. Quant à l'augmentation du taux de forêt dense, contraire aux résultats de Tshibangu (2001), elle pourrait être liée à la composition de la classe "forêt». En effet, pour son étude, sous l'appellation "forêt", il a combiné ce que nous avons dénommé "forêt dense » et «forêt secondaire ". L'augmentation du taux de forêt dense est une bonne chose pour la conservation; même si ces forêts nouvellement formées sont plutôt constituées de fragments isolés et de petites tailles, caractérisés par un effet de lisière considérable (Bogaert et al., 2000).

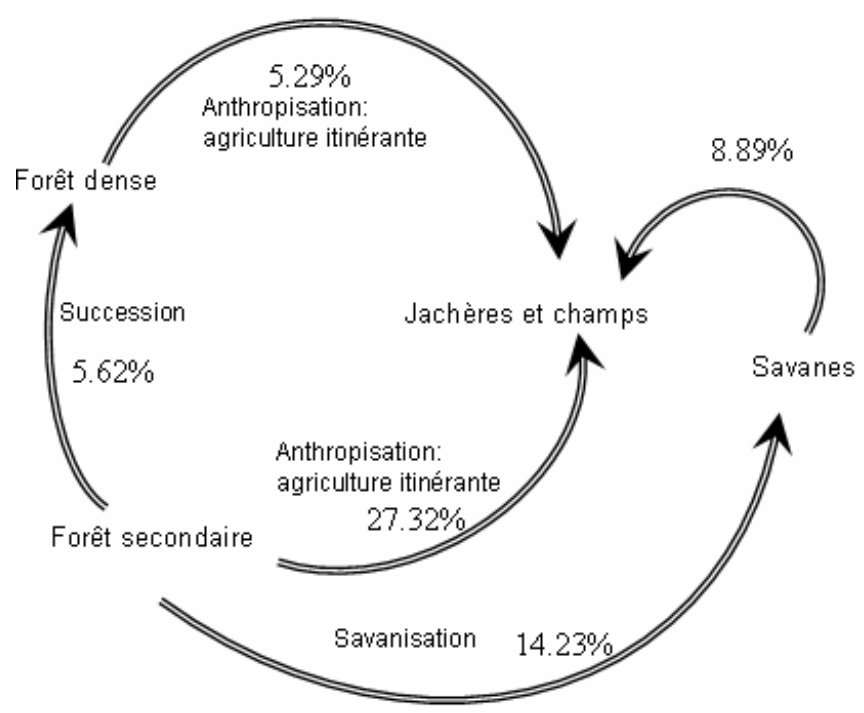

Figure 5 : Principaux taux de transformations opérés dans les classes d'occupations du sol entre 1960 et 2005. 


\subsection{Correspondance entre les changements détectés par les cartes et les indices de structure spatiale}

Le calcul des indices de structure spatiale a permis de mettre en évidence la configuration spatiale des taches des classes dans le paysage. L'analyse cartographique, grâce à la matrice de transition, nous a révélé trois processus qui se sont déroulés entre 1960 et 2003. Premièrement, la savanisation matérialisée par l'augmentation de l'aire totale des savanes et la formation de nouvelles savanes dans le paysage, ensuite la dégradation de l'écosystème forestier et enfin une faible tendance d'évolution écologique vers un état climacique (formation de forêts denses). Les indices de structure spatiale ont aussi révélé un certain nombre de transformations spatiales opérées dans notre paysage en 45 ans. Mais on constate que pour l'ensemble des indices calculés, les processus de transformation sont beaucoup plus amplifiés dans les classes des forêts denses et secondaires que dans les classes des savanes et des jachères et champs. En plus, les trois processus révélés par la cartographie sont confirmés par les indices de structure spatiale. La dégradation de l'écosystème forestier est matérialisée par une fragmentation plus forte au niveau de ces classes. Cela est illustré par une forte augmentation du nombre de taches pour les forêts secondaires et forêts denses ainsi que par une diminution aussi forte de l'aire moyenne des taches. Quant à l'indice de dominance, pendant qu'elle s'accroît dans les classes savanes et jachères et champs, elle décroît dans les deux classes forestières. Aussi, la savanisation est matérialisée par l'augmentation de l'aire totale des savanes, l'augmentation de la dominance de la tache la plus grande de cette classe et la constance de l'hétérogénéité (indice de Shannon). La question fondamentale que l'on pourrait se poser est de savoir si les résultats obtenus dans le calcul des indices n'ont pas été influencés par les niveaux de génération cartographique. II est bien connu que la complexité de la structure des données complique l'analyse et empêche souvent la combinaison de cartes de sources différentes (Bogaert \& Mahamane, 2005). Les échelles initiales des deux cartes utilisées étant différentes, les niveaux de détails seront aussi différents. Cela pourrait avoir une répercussion sur les indices spatiaux calculés. D'où la nécessité d'atténuer au préalable ces différences par I'utilisation de techniques comme celle du Minimum Mapping Unit (Saura, 2002). Cet effet de la cartographie serait beaucoup plus marqué dans le calcul du nombre de taches, le périmètre et les indices qui utilisent ces valeurs, comme l'indice des formes (Farina, 1998). En effet les données matricielles sont exprimées par des pixels alors que les données vectorielles utilisent des points, lignes ou polygones pour les représentations cartographiques. C'est donc logique que les pixels de par leur résolution influenceront les formes des objets pendant la classification (McGarigal \& Marks, 1995). Mais, cela ne saurait expliquer les amplitudes très variables observées entre les différentes classes ni au niveau des aires moyennes $\bar{a}_{j}$ ni au niveau des dominances $D_{j}$ (a). En effet, nous avons constaté que sur une même carte, les plus grands taux de variation de la quasitotalité des indices sont observés spécifiquement au niveau des forêts comparativement à la savane et aux jachères et champs. Les amplitudes de la dynamique spatiale sont plus fortes dans les classes des forêts. En plus, les tendances observées entre les deux groupes de classes (groupe des forêts et le groupe des savanes, jachères et champs) évoluent en sens opposé. En définitive, les résultats obtenus reflètent la réalité de la tendance de la dynamique du paysage dans cette zone du Bas-Congo. Sinon on aurait dû avoir les mêmes proportions de variations et taux de changements au niveau des indices pour toutes les classes d'une même carte.

\section{Conclusion}

La présente étude a permis grâce à la matrice de transition appuyée par le calcul d'indices de structure spatiale, de quantifier les changements opérés dans un paysage rural situé dans la province du Bas-Congo en R.D. Congo. Trois grandes transformations y ont été identifiées, à savoir la dégradation de l'écosystème forestier, la savanisation et une faible tendance de succession écologique végétale naturelle. Ces changements sont principalement dus à des perturbations d'origine anthropique. En effet, la pression démographique et les pratiques agricoles non durables ont contribué à la modification de l'occupation du sol. La configuration spatiale du paysage a profondément changé en 45 ans. La matrice du 
paysage est passée de forêt secondaire aux jachères et champs. Plus de $41 \%$ du paysage constitué par la forêt secondaire en 1960 s'est transformé, soit en jachères et champs, soit en savanes. Cela constitue une inquiétude pour la restauration de l'écosystème où la forêt secondaire occupe une place centrale. Pour donc contribuer à conserver la biodiversité via I'habitat, une sensibilisation des populations s'impose. D'abord pour la création et le développement de forêts secondaires villageoises, gage de la gestion durable des ressources naturelles et dans lesquelles les populations pourront tirer des produits forestiers pour leurs besoins usuels. Ensuite par l'amélioration du système agraire archaïque très «dévoreur et gaspilleur des terres» au profit d'un système d'agroforesterie ou encore de l'assolement, procédure de culture intensive et plus économique du point de vue de la superficie (Tshibangu, 2001).

\section{Remerciements}

Les auteurs remercient le gouvernement et surtout le contribuable de la Côte d'Ivoire pour nous avoir octroyé la bourse nécessaire à cette étude et les personnes ci-après citées : $E$. WOLFF et $A$. CAREER (IGEAT, ULB), J. LAVREAU (Muséum Royal d'Afrique Centrale), C. VANCUTSEM et P. DUFOURNY (UCL), C. POTVLIEGE et D. BAUWENS (Service Laïque de Coopération au Développement).

\section{Références citées}

Bizangi K., 2004. Impact de la production des combustibles ligneux en RDC cas du Katanga, de Kinshasa et du Bas-Congo. In: Acte des séminaires de formation et ateliers de haut niveau en évaluation environnementale. 12-17 Janvier 2004. Kinshasa (R.D. Congo): Association Nationale pour l'Evaluation environnementale (ANEE), pp. 105-119.

Bogaert J. \& Mahamane A., 2005. Ecologie du paysage: cibler la configuration et l'échelle spatiale. Annales des Sciences Agronomiques du Bénin (7) 1 : 39-68.

Bogaert J., Rousseau R. , Van Hecke P. \& Impens I., 2000. Alternative area-perimeter ratios for measurement of 2D shape compactness of habitats. Applied Mathematics and Compilation 111 : 71-85.
Burel F. \& Baudry J., 2003. Ecologie du paysage. Concepts, méthodes et applications. Paris, France : Tec \& Doc. 359 pp.

Compère P., 1970. Carte des sols et de la végétation du Congo, du Rwanda et du Burundi. 25. Bas-Congo. B. végétation. I.N.É.A.C., Office nationale de la recherche et du Développement au R.D. Congo. Bruxelles, Belgique. 35 pp.

Davidson C., 1998. Issues in measuring landscape fragmentation. Wildlife Soc. Bul. 26 : 32-37.

Delcros P., 1994. Ecologie du paysage et dynamique végétale post-culturale. Thèse de doctorat, Université Joseph Fourier, Grenoble I, France. 269 pp.

Ducrot D., 2005. Méthodes d'analyses et d'interprétation d'images de télédétection multisource. Extraction de caractéristiques du paysage. Mémoire de recherche. INP, Toulouse, France. $216 \mathrm{pp}$.

Duvigneaud P., 1949. Les savanes du BasCongo. Essai de phytosociologie topographique. Révue Botanique, Lejeunia. Déc. 1949, Liège. Mém. 10 :192 pp.

FAO, 2001. FOSA Document national de prospective - République Démocratique du Congo http://www.fao.org/docrep/003/X6779F/ X6779F00.HTM

Farina A., 1998. Principles and Methods in Landscape ecology. London, UK: Chapman \& Hall. 235 pp.

Fortin M. J., 2002. Analyse spatiale en écologie: problèmes statistiques et de mise à l'échelle au niveau du paysage. Ecoscience 9 : iii-v.

Hargis C. D., Bissonnette J. A. \& David J. L., 1997. Understanding measures of landscape pattern: 231-261. In : Bissonnette J. A. (ed.). Wildlife and landscape ecology. Springer, New York.

Kio O. P. R., 1984. Stratégie de conservation des forêts en Afrique Tropicale. Compte Rendu de colloque du 27 Avril au 01 Mai 1981 à Ibadan, Nigéria. 188 pp.

Krummel J. R., Gardner R. H., Sugihara G., O'Neill R. V. \& Coleman P. R., 1987. Landscape pattern in a disturbed environment. Oikos 48: 321-324.

McGarigal K. \& Marks B. J., 1995. Fragstats: Spatial Pattern Analysis Program for Quantifying 
Structure. Department of Agriculture, Pacific Northwest Research Station General Technical Report PNW-GTR-351. Oregon, USA. (disponible sur Internet http: // www.fs.fed.us/pnw/pubs/ gtr_351.pdf)

OIBT, 2002. Réintégrer les forêts secondaires dans le paysage. OIBT Actualités des Forêts Tropicales 10/4 2002.

Ozenda P., 1986. La cartographie écologique et ses applications. Masson, Paris, France. 160pp.

Roche P., 1998. Dynamique de la biodiversité et action de l'homme. Rapport ENV-SRAE - 94233, Paris, France. 6 pp.

Saura S., 2002. Effects of minimum mapping unit on land cover data spatial configuration and composition. Int. J. Remote sensing 23: 4853-4880.
Schlaepfer R., 2002. Analyse de la dynamique du paysage. Fiche d'enseignement 4.2, Laboratoire de Gestion des Ecosystèmes, Ecole Polytechnique de Lausanne, Suisse. 10 pp.

Tshibangu K. W. T., 2001. Etude du déboisement et de la crise de combustibles ligneux en tant que source d'énergie domestique à Kinshasa (République Démocratique du Congo). Thèse de doctorat. Université Libre de Bruxelles, Belgique. $274 \mathrm{pp}$.

Vink A. P. A., 1983. Landscape ecology and land use. Longman, New York, USA. 264 pp.

Whitmore T. C., 2005. An Introduction to Tropical Rain Forests. Oxford University press, New York, USA. 296 pp.

Wolff E., 2005. Rapport de mission cartographique à Kisantu (R.D. Congo) du 15 au 30 Septembre 2005. SLCD, Bruxelles, Belgique. 9 pp. 